\title{
Guidelines for perioperative antithrombotic therapy in hair restoration surgery: management of patients with coronary heart disease, mechanical heart valve, and atrial fibrillation
}

\author{
Kuniyoshi Yagyu, MD Tokyo, Japan nhttkyo11@kioicho.jp
}

\section{Introduction}

Perioperative antithrombotic therapy is an important issue to avoid cardiovascular events during surgery in a patient with coronary heart disease or atrial fibrillation. Usually, anticoagulant and antiplatelet drugs are stopped for 5-10 days before hair restoration surgery. However, interruption of antithrombotic drugs may cause thrombosis resulting in serious cardiovascular events. Recently, guidelines on perioperative management of antithrombotic therapy have changed. Updated guidelines recommend that patients do not stop these drugs before surgery.

Is hair restoration surgery possible if antithrombotic drugs cannot be stopped before surgery? Is there any way to perform a safe hair transplant session without major bleeding in patients under antithrombotic therapy? The answer is yes, and this article will describe the way for safe surgery.

The author has referred to the guidelines of several societies, which include the American Heart Association, the American College of Cardiology Foundation, the European Society of Cardiology Committee, the Japanese Circulation Society, etc., and will describe updated guidelines on perioperative antithrombotic therapy for patients with coronary artery disease, mechanical heart valve, and atrial fibrillation. The following guidelines will allow a physician to perform a safe operation even in patients with cardiovascular diseases.

\section{Patients}

This study included 25 male patients with cardiovascular disease who underwent 46 hair transplant operations. Patient mean age was $54.9 \pm 11.0$ years. One to four sessions using a total of about 1,400 to 4,800 grafts were performed. The disorders included coronary heart disease in 28 operations, ischemic cerebral disease in 7, valvular heart disease in 2, and pulmonary embolism in 1. Atrial fibrillation in 8 operations included permanent atrial fibrillation in 4 , and paroxysmal atrial fibrillation and flutter in 4. Ischemic heart disease included angina pectoris, unstable angina, and myocardial infarction. Coronary artery stenting treatment and percutaneous coronary intervention had been performed in 9 , and coronary artery bypass graft surgery with percutaneous coronary intervention in 2 .

Among these, the following were operations in patients under maintenance antithrombotic therapy: coronary heart disease in 10 operations, ischemic cerebral disease in 7, atrial fibrillation in 4, valvular heart disease in 1, and pulmonary embolism in 1 .

Risk factors in these patients were coronary artery stents, mechanical prosthetic heart valve, atrial fibrillation, and systemic arterial sclerosis including stenosis in the coronary artery and cerebral artery. Possible complications related to thrombosis were acute myocardial infarction, stent thrombosis, mechanical prosthetic heart valve thrombosis, and acute ischemic stroke.

\section{Results}

All patients went through safe operations without cardiovascular events. Monitors of precordial electrocardiogram, pulse oximeter $\left(\mathrm{SpO}_{2}\right)$, blood pressure, and heart rate were essential for safe operation in every case. Prophylactic usage of a nitroglycerin tape was helpful in preventing ischemic heart attack in patients with ischemic heart disease and in patients over 60 years of age.

Dosage of antithrombotic drugs was controlled following the guidelines in this article. Anticoagulant drug and antiplatelet drugs were continued before surgery as were all the other maintenance drugs, including beta blocker. Epinephrine in tumescence solution was used as usual or in lower dose. There was no hemorrhagic tendency during the hair transplantation procedure.

\section{Presentation of Cases}

The first case was a 50-year-old male with type VI male pattern hair loss. In his past history, he had hypertension and hyperlipidemia. He experienced acute myocardial infarction at age 49 , and he had undergone percutaneous coronary intervention treatment with coronary artery stent implantation (Figure 1). He visited our clinic for hair transplantation 1.5 years after the stent implantation. Computerized tomographic angiogram showed coronary artery stent at the proximal portion in the left anterior descending coronary artery (Figure 2).

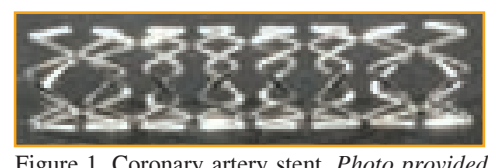

Figure 1. Coronary artery stent. Photo provided courtesy of St. Jude Medical Co. in Japan.

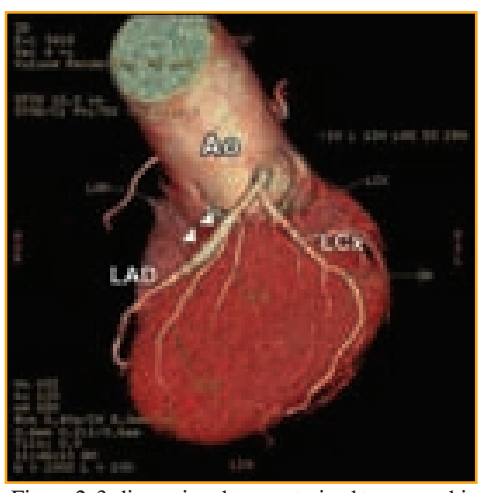

Figure 2. 3-dimensional computerized tomographic angiogram of a patient with coronary stent implantation. White triangles indicate coronary artery stent in the left anterior descending coronary artery. Abbreviation: Ao, aorta; LAD, left anterior descending artery; LCx, left circumflex artery.
In this particular patient, the risk factors were myocardial infarction, coronary artery stent, and systemic arterial sclerosis including stenosis in the coronary artery and cerebral artery. Possible complications during surgery were stent thrombosis, acute myocardial infarction, and cerebral infarction. In order to prevent these complications, low blood pressure, hypoxemia, and emotional distress were avoided during surgery.

As for the maintenance drugs, 100mg aspirin and antihypertensive drugs including beta blocker, calcium channel blocker, and angiotensin II receptor blocker were continued in the maintenance dosage before surgery. Nitroglycerin tape was used for coronary vasodilation during the procedure. Fentanyl and midazolam were given as a sedative and an analgesic. Hemodynamic parameters were stable during the operation.

Four sessions were performed successfully from age 50 to 53 without any complication. Low-dose aspirin did not cause hemorrhagic tendency during surgery. A total of 4,845 grafts were transplanted from the front to the vertex areas.

Case 2 was a 45-year-old male with type Va male pattern hair loss. He had suffered from aortic valve regurgitation and annulo-aortic ectasia, and he underwent cardiac surgery by Bentall operation 9 years before. The surgical technique involved

$\Rightarrow$ page 60 
Guidelines for management

from page 59

replacement of the aortic valve, aortic root, and ascending aorta with a composite graft using mechanical prosthetic heart valve and vascular prosthesis, and the coronary arteries were reimplanted into the vascular graft.

The patient required antithrombotic therapy for the mechanical heart valve (Figure 3). He took 4mg warfarin and $100 \mathrm{mg}$ aspirin every day as anticoagulant and antiplatelet drugs. Other maintenance drugs included digoxin $(0.1 \mathrm{mg}$ metildigoxin) as inotropic drug, beta blocker (2.5mg bisoprolol) for tachycardia, 100mg fenofibrate for hyperlipidemia, and $10 \mathrm{mg}$ histamine $\mathrm{H}_{2}$ receptor

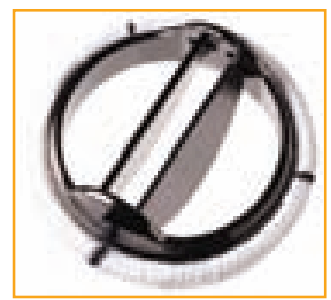

Figure 3. An example of a mechanical prosthetic heart valve. Photo provided courtesy of St. Jude Medical Co. in Japan. antagonist (famotidine) for stomachache. The results of blood tests before surgery were almost normal including platelet count 203,000 per microliter of blood. Prothrombin time was 21.8 seconds and the international normalized ratio of prothrombin time was 1.95 .

Dosage of warfarin was reduced to $2.5 \mathrm{mg}$ per day for 3 days before the hair transplantation. Warfarin was increased to $4 \mathrm{mg}$ per day in the evening 1 day before the surgery and the maintenance dose of warfarin was continued after the surgery. All the other maintenance drugs were continued in the same dosage before the session including aspirin, digoxin, and beta blocker.

A nitroglycerin tape was put on the patient's chest skin to avoid cardiac ischemia during surgery. There was no significant change of ST segment on the electrocardiogram during the surgery.

Hair transplantation was performed safely without any complication, and 1,917 grafts were implanted from the front to the mid-scalp areas. There was no hemorrhagic tendency during surgery.

\section{Other Cases}

There were 8 operations in patients with permanent atrial fibrillation and paroxysmal atrial fibrillation and flutter that were performed safely without any complication. Maintenance drugs including low-intensity warfarin, low-dose aspirin, antiarrhythmic drugs, coronary vasodilator, antihypertensive drugs, beta blockers, sedatives, and other drugs were continued before surgery. There was no hemorrhagic tendency during surgery.

\section{Discussion}

Perioperative control of antithrombotic drugs is an important issue for safe operation in patients with cardiovascular diseases under antithrombotic therapy. Antithrombotic therapy consists of two classes of drugs. They are anticoagulant drugs and antiplatelet drugs. Anticoagulant drugs reduce fibrin formation and slow down clotting. Antiplatelet drugs prevent platelets from clumping. A combination of anticoagulant drugs and antiplatelet drugs is important to prevent clot formation and growth.

Antithrombotic therapy. Control of antithrombotic therapy is crucial in patients with cardiovascular disease. An antiplatelet drug is important for prevention of stent thrombosis in patients after coronary stent implantation and is effective for the prevention of stroke in patients with atrial fibrillation. A combination of anticoagulant drugs and antiplatelet drugs is of critical importance for prevention of prosthetic valve thrombosis in patients after replacement of the heart valve using mechanical heart valve.

If you want to operate on a patient under antithrombotic therapy, you should talk to the patient's physician about control of these medicines. If the cardiologist doesn't mind stopping the antithrombotic drugs for several days, antithrombotic drugs can be stopped before surgery and restarted after the surgery. If the cardiologist doesn't allow you to stop antithrombotic drugs, the drugs should be continued before the procedure. Anyway, hair transplantation is safe operation with low risk of bleeding.

Risk of bleeding vs. risk of thrombosis. We should always compare the risk of bleeding and the risk of thrombosis in cardiovascular patients. There are some diseases that require lifelong antithrombotic therapy in order to prevent cardiovascular events caused by thrombosis. Disorders requiring lifelong antithrombotic therapy are classified into two groups: low-risk and high-risk.

Disorders in the low-risk group include old myocardial infarction, old cerebral infarction, permanent atrial fibrillation, bioprosthetic heart valve, and deep vein thrombosis. In these patients, thrombosis does not cause serious cardiovascular events, and antithrombotic drugs can be reduced to a half dose or they can be stopped for a few days before surgery.

Disorders in the high-risk group include unstable angina pectoris, recent cerebral infarction, acute phase of transient ischemic attack, and patients with mechanical prosthetic heart valve and coronary artery stent. In these patients, thrombosis may cause serious cardiovascular events, and antithrombotic drugs should be continued in the maintenance dose or they can be reduced to two-thirds dose for only a few days before the hair transplantation. If you stop antithrombotic drugs before surgery, the patient will be put at risk of thrombosis with high mortality.

Risk of thrombosis in cardiac patients. Special attention should be paid to prevent thrombosis in a patient with a coronary artery stent and mechanical heart valve. Stent thrombosis and valve thrombosis result in a severe complication with high mortality. Coronary stent thrombosis causes acute myocardial infarction with $20 \%$ mortality within one year. Risk of non-fatal myocardial infarction will increase if you stop low-dose aspirin $(75-300 \mathrm{mg} /$ day), which is used for prevention of a secondary cardiovascular event in patients after myocardial infarction or in patients with diabetes. ${ }^{1,2}$

Mechanical prosthetic heart valve thrombosis results in stuck valve with malfunction of the valve (Figure 4). Stuck valve cannot close or open completely, and it causes severe regurgitation and stenosis of the valve and hemolytic anemia, which cause severe acute heart failure. The patient requires emergency reoperation to replace the malfunctioned heart valve. The redo open heart surgery is sometimes challenging with high operative risk and mortality. Thrombus

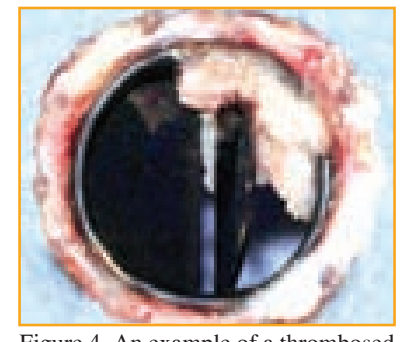

Figure 4. An example of a thrombosed mechanical heart valve. Stuck valve cannot open or close completely, which causes severe stenosis and regurgitation of the valve and results in severe acute heart failure requiring reoperation with high mortality. formed on the metallic surface of the mechanical heart valve may be detached and it may result in thromboembolism. The size of the thrombus detached from the mechanical heart valve is usually big, and it occludes large branch of the cerebral artery, which results in severe acute cerebral infarction in a wide area.

These are the reasons why the patients with high risk of thrombosis need prevention of blood clot formation. Continuation of antithrombotic drugs and prevention of acute thrombosis is crucial for safe hair transplant surgery in these patients.

Use of low-dose aspirin (160-325mg/day) is recommended 
as class I indication on the level of evidence $\mathrm{A}$ in order to reduce recurrence and mortality rate in acute phase and chronic phase after atherosclerotic cerebral infarction, lacuna infarction, and cardiogenic thromboembolism (American Heart Association/American Society of Anesthesiologists guideline 2007, European Stroke Organization guideline for ischemic stroke 2008, and Japanese Circulation Society guideline of treatment for stroke 2009). These guidelines suggest that the rate of recurrence and mortality will increase both in the acute and chronic phase after stroke if you stop low-dose aspirin.

Coronary heart disease. Coronary heart disease caused about 1 of every 6 deaths in the United States in 2007. Each year, an estimated 785,000 Americans will have a new coronary attack, and about 470,000 will have a recurrent attack. It is estimated that an additional 195,000 silent first myocardial infarctions occur each year. ${ }^{3}$ Estimation by the National Health Interview Survey and the National Center for Health Statistics in 2008 reported that prevalence of coronary artery disease for people over 18 years of age was about $6 \%$, and the possibility is about $47 \%$ in patients over 60 years of age. These data suggest that 1 of every 17 patients in your daily practice may have coronary heart disease, and the possibility is higher in elderly patients.

Coronary artery stent. The diagnosis and treatment of coronary heart disease is made by percutaneous coronary intervention, known as coronary angioplasty, and stenotic segments of the coronary artery are treated using a coronary artery stent of which there are two types - the bare metal stent and drug eluting stent. The bare metal stent is a conventional vascular stent without coating. It may cause neointimal hyperplasia resulting in restenosis of the coronary artery within 6 months after the initial percutaneous coronary intervention treatment.

The drug eluting stent is a new device with coating that prevents cell proliferation and fibrosis in the coronary artery. The drug eluting stent has dramatically reduced the incidence of restenosis after percutaneous coronary intervention. However, a polymer on the drug eluting stent is recognized as a foreign substance, and it induces hypersensitivity reaction and eosinophilic infiltration in the local vessel wall. A drug eluting stent inhibits vascular smooth muscle proliferation and delays re-endothelialization, which increases the risk of stent thrombosis and occlusion. ${ }^{4}$ Stent thrombosis frequently results in serious complications including myocardial infarction and cardiac death. Very late stent thrombosis is one of the greatest concerns more than 1 year after drug eluting stent implantation. Discontinuation of antiplatelet drugs results in very late stent thrombosis, which occurs even 3 years after stent implantation. Incidence of stent thrombosis within 3 years increases at a rate of $0.4-0.6 \%$ per year and $1.2-2.9 \%$ at 3 years. ${ }^{5}$

Antithrombotic drugs and coronary stents. The possibility of restenosis and occlusion of the coronary artery was 20-30\% with the bare metal stent but less than $20 \%$ with the drug eluting stent at 5 years after stent implantation. Patients with bare metal stents require antiplatelet drugs for up to 12 months. On the contrary, a drug eluting stent has the possibility to cause late local thrombosis and the patients require antiplatelet drugs for more than 3 years.

Bare metal stents and antithrombosis. In order to prevent coronary artery stent thrombosis, antiplatelet drugs should be used for 1-6 months in patients with a bare metal stent. Hair transplantation operation within 1 month after stenting should be postponed, because antiplatelet drugs should not be stopped in this period.
In patients more than 6 months after stent implantation using a bare metal stent, antiplatelet drugs can be stopped for a few days or reduced to a half dose safely for one week. Safe operation is possible in these patients.

Drug eluting stents and antithrombosis. Two types of antiplatelet drugs should be continued within 12 months after stent implantation using the drug eluting stent. Usually, a combination of aspirin $(75-300 \mathrm{mg} /$ day) and clopidogrel $(75 \mathrm{mg} /$ day) is prescribed for 1 year. Antiplatelet drugs should not be stopped, and hair transplantation should be postponed until 1 year after the stenting in these patients.

In patients more than 1 year after the stent implantation using a drug eluting stent, two types of antiplatelet drugs are usually continued. In these patients, hair transplant session is possible. You can stop clopidogrel for 5 days as long as you continue aspirin before surgery. If you want to stop both drugs before surgery, you can stop them for only 3 days, but not longer. After the hair transplantation, both drugs should be restarted.

In patients more than 2 years after stent implantation using a drug eluting stent, one antiplatelet drug (aspirin or clopidogrel) is continued in the long-term. You can stop aspirin for 3-5 days, or low-dose aspirin can be continued before surgery. Low-dose aspirin does not cause hemorrhagic tendency, and it can be continued before surgery. Safe hair transplantation is possible in these patients. Antiplatelet drugs should be restarted on the next day after the hair transplantation.

As for operative risk and coronary artery disease, patients after coronary stent implantation and coronary artery bypass graft operation are safer than patients before percutaneous coronary intervention, because coronary blood flow is secured.

As for the place of stenosis in the coronary artery, stenosis in the left main trunk and the left anterior descending artery is very dangerous, whereas stenosis in the right coronary artery and the left circumflex artery is not dangerous. Occlusion of the left anterior descending artery will result in acute myocardial infarction in the anteroseptal wall of the left ventricle, which results in severe heart failure with high mortality. Occlusion of the right coronary artery or left circumflex artery will result in acute myocardial infarction in the inferior wall or lateral or posterior wall of the left ventricle, which results in acute heart failure with low mortality.

Atrial fibrillation. Atrial fibrillation is the most common sustained cardiac rhythm disturbance. The prevalence of atrial fibrillation is estimated at $0.4 \%$ of the general population, increasing with age and it occurs in more than $6 \%$ of those over 80 years of age.

Atrial fibrillation is commonly classified into three types: paroxysmal atrial fibrillation, persistent atrial fibrillation, and permanent atrial fibrillation. Paroxysmal atrial fibrillation means recurring sudden episodes of atrial fibrillation. Each episode of atrial fibrillation comes suddenly, and arrhythmia stops suddenly within 7 days. The heart beat becomes normal sinus rhythm again. Duration of each episode may vary from several minutes to several days. Symptoms of arrhythmia include palpitation, breathlessness, and dizziness. Persistent atrial fibrillation means that atrial fibrillation may last longer than 7 days. The arrhythmia can revert to normal sinus rhythm after treatment by cardioversion. About half of the patients will revert back to atrial fibrillation within one year. Permanent atrial fibrillation means that it is longstanding arrhythmia and the heart rhythm does not revert back to normal sinus rhythm. Patients with persistent atrial fibrillation and permanent atrial fibrillation usually have no symptoms.

$\Rightarrow$ page 62 
Guidelines for management

from page 61

One of the possible complications of atrial fibrillation is an increased risk of having a stroke. Loss of organized mechanical contraction of the atrial wall causes reduced flow velocity and thrombus formation in the left atrium and most frequently in the left atrial appendage.

A clot in the left atrium does not usually peel off the wall in patients with permanent atrial fibrillation. Permanent atrial fibrillation is relatively safe and the risk of systemic thromboembolism is low. You can reduce dosage of an anticoagulant drug or you can stop it before surgery, if you want, in patients with permanent atrial fibrillation.

On the contrary, paroxysmal atrial fibrillation is sometimes dangerous. When the paroxysmal atrial fibrillation reverts to normal sinus rhythm, the fibrillated left atrial appendage begins to contract again. It is the most dangerous period, when a newly formed unstable thrombus may peel off the atrial wall. The clot travels in the systemic circulation causing systemic thromboembolic events and acute cerebral infarction. In patients with paroxysmal atrial fibrillation, strict antithrombotic therapy is mandatory and the antithrombotic drugs should be continued before surgery.

Atherosclerotic occlusion of the cerebral artery usually occurs at the peripheral cerebral artery, which results in cerebral infarction in a small area. On the contrary, the size of thrombus detached from the atrial wall is big in patients with atrial fibrillation, which occludes a large branch of the cerebral artery resulting in cerebral infarction in a wide area. This is the reason why the brain damage caused by atrial fibrillation is often more serious than that of an ordinary stroke caused by atherosclerosis.

According to the updated guidelines on antithrombotic therapy for atrial fibrillation, anticoagulant therapy using warfarin or dabigatran is recommended (Class I recommendation) ${ }^{5-7}$ Meta-analysis showed that adjusted-dose oral anticoagulation is highly efficacious for prevention of all stroke (both ischemic and hemorrhagic), with a risk reduction of $61 \%$ versus placebo. The most common anticoagulant drug is warfarin, which reduces the risk of a stroke by nearly two-thirds. Maximum protection against ischemic stroke in atrial fibrillation is achieved with the international normalized ratio of prothrombin time of 2.0-3.0..$^{9,10}$ The international normalized ratio range of 1.6-2.6 is effective in patients over 70 years of age. Anticoagulant therapy using warfarin can be continued before tooth extraction and minor surgery including hair transplantation. In patients with atrial fibrillation without mechanical heart valves, anticoagulation can be interrupted for a period of up to 1 week before the procedures with some increased risk of ischemic stroke.

Effectiveness of antiplatelet therapy for patients with atrial fibrillation is controversial. Aspirin offers only modest protection against stroke for patients with atrial fibrillation. The effect is less consistent than that of oral anticoagulation (Class IIa recommendation). ${ }^{9}$ Antiplatelet therapy is not mandatory by some guidelines for prevention of ischemic stroke in patients with atrial fibrillation. ${ }^{9-11}$

Bleeding from slits. Hair transplant surgeons may be afraid of bleeding during surgery, and they want to stop antiplatelet drugs and anticoagulant drugs before surgery. Bleeding during surgery and antithrombotic drugs are different issues. Bleeding from slits can be reduced by intradermal injection of tumescent solution containing a small amount of epinephrine. If the tumescence is injected correctly into the dermal layer around the recipient area, there will be little bleeding from slits, because most of blood flow comes through the dermal layer and it can be reduced remarkably by vasoconstriction after intradermal injection of tumescent solution with epinephrine. The dilution of epinephrine can be reduced to as low as 1:400,000 to 800,000 in the tumescence. Only low concentration of epinephrine is effective to reduce bleeding from skin incisions. Injection of a high concentration epinephrine solution, such as super juice, to promote hemostasis is not necessary, if you use this technique of intradermal injection of tumescence.

If you inject tumescence in the dermal layer correctly, the skin color in the injection site will become pale white. If the skin color doesn't change, it means that the injection is too deep and the tumescent solution was injected into the subcutaneous layer under the dermis, which does not reduce bleeding from slits. If some bleeding from slits still remains, to stop it you should use gauze to compress the slits for a few minutes while making slits in other recipient areas.

Bleeding from skin incision during donor harvesting can also be minimized by injecting tumescent solution in the dermal layer all around the donor skin incision. Typically during donor strip harvesting, most of the blood flow comes through the dermal layer, and there is little bleeding from the subcutaneous adipose tissue. After the donor skin incision but before donor harvesting, we can see the exact places of bleeding in the dermal layer. This allows us to inject additional tumescent solution only around these areas to increase tissue pressure around the small vessels and to contribute to haemostasis.

Pharmacokinetics of antiplatelet drugs. Aspirin, ticlopidine, clopidogrel, dipyridamole, and cilostazol are usually used as antiplatelet drugs. The effect of aspirin, ticlopidine, and clopidogrel is irreversible, while the effect of dipyridamole and cilostazol is reversible (Table 1).

A normal count of platelets is about $150,000-400,000$ per microliter of adult peripheral blood. The average lifespan of a normal circulating platelet is about 5-10 days. If the number of platelets with normal adhesiveness is more than 50,000 per microliter, general surgery, orthopedic surgery, and even cardiac surgery can be performed safely without hemorrhagic tendency.

If aspirin or clopidogrel is stopped for 5-7 days before surgery, more than a half of platelets in the peripheral blood become renewed platelets with normal function on the day of the surgery, and safe hair transplantation will be possible even if the effect of aspirin and clopidogrel is irreversible. It may, however, be dangerous if you stop aspirin or clopidogrel for 10-14 days, because almost all the platelets become normal again with normal clotting function.

Aspirin inhibits cyclooxygenase- 1 enzyme and it inhibits production of thromboxane A2. The irreversible effect of aspirin lasts for the lifetime of the platelet. High-dose aspirin $(1,000-$ $1,200 \mathrm{mg} /$ day), ticlopidine, and clopidogrel can be stopped for about 7 days before surgery. Low-dose aspirin (75-300mg/day) does not cause hemorrhagic tendency. If aspirin is the only antiplatelet drug for the patient, we can perform the hair transplant session without major bleeding tendency even if low-dose aspirin is continued. The antiplatelet action of aspirin is mild in comparison with other antiplatelet drugs.

\begin{tabular}{|lll|}
\hline Antiplatelet Drug & Effect & Half-life \\
aspirin & irreversible & \\
ticlopidine & irreversible \\
clopidogrel & irreversible & \\
icosapentate & reversible & 326 hours \\
dipyridamole & reversible & 25 minutes \\
cilostazol & reversible & 18 hours \\
beraprost (PG I2) & reversible & $45-57$ minutes \\
limaprost (PG E2) & reversible & 27 minutes \\
\hline The average life span of a platelet is normally 5 to 10 days. PG: prostaglandin \\
\hline \multicolumn{2}{c}{ Table 1. Effect and half-life of antiplatelet drugs } \\
\hline
\end{tabular}


Ticlopidine (Ticlid) (200-300mg/day) and clopidogrel (Plavix) $(75 \mathrm{mg} /$ day) inhibit adenosine diphosphate chemoreceptors on platelet cell membranes. These prevent the conformational change of glycoprotein IIb/IIIa, which allows platelet aggregation and binding to fibrinogen. The irreversible effect of these drugs lasts for the lifespan of a platelet.

Dipyridamole (200-300mg/day) inhibits platelet phosphodiesterase and adenosine reuptake, causing an increase in cyclic AMP with potentiation of release and the action of prostaglandin $\mathrm{I} 2$, which opposes actions of thromboxane A2. A half-life of dipyridamole is about 25 minutes in the blood. If you stop dipyridamole for 1 day, platelet aggregation activity will become normal again. Cilostazol (200mg/day) selectively inhibits phosphodiesterase and increases cyclic AMP. The half-life of cilostazol is 18 hours in the blood. Dipyridamole and cilostazol can be continued in the maintenance dose or they can be stopped for one day before surgery without hemorrhagic tendency during the procedure.

Pharmacokinetics of anticoagulant drugs. Warfarin (Coumadin) is the most widely prescribed oral anticoagulant drug and it is a vitamin $\mathrm{K}$ antagonist, so it inhibits vitamin K epoxide reductase and vitamin K-dependent synthesis of the calcium-dependent clotting factors VII, IX, and X in the liver. Recent evidence suggests that the anticoagulant and antithrombotic effects of warfarin can be dissociated and that reduction of prothrombin and possibly factor $\mathrm{X}$ are more important than reduction of factors VII and IX for the antithrombotic effect. ${ }^{12}$ The half-life of warfarin is about 36 hours in the blood. The anticoagulant effect of warfarin appears in 12-24 hours and lasts for 48-72 hours. It takes several days for warfarin to reach the therapeutic effect, and warfarin remains effective for several days after it is stopped. The anticoagulant activity of warfarin is closely monitored by the international normalized ratio of prothrombin time to ensure that an adequate and safe dose is taken. The targeted international normalized ratio level tends to be 2.0-3.0 in most conditions as maintenance anticoagulant therapy for prevention of thrombosis.

Interruption of anticoagulation before surgery can be problematic. If warfarin is stopped 4-5 days pre-operatively, the international normalized ratio will return to normal $(<1.2)$ at the time of the procedure, ${ }^{12}$ and the patients remain unprotected for about 2-3 days post-operatively. The period off warfarin can be reduced by restarting warfarin in the maintenance dose 1-2 days before the procedure with the expectation that the international normalized ratio will return to normal at the time of the procedure and warfarin will become effective again soon after the surgery. ${ }^{12}$

For patients at low risk of thrombosis (e.g., permanent atrial fibrillation), warfarin can be reduced to half of the maintenance dose for 4-5 days in advance of surgery to allow the international normalized ratio to fall to normal or near normal (1.3-1.5). The maintenance dose of warfarin is resumed post-operatively. ${ }^{12}$

For patients at high risk of thrombosis (e.g., mechanical heart valve), the dose of warfarin can be reduced to two-thirds of the maintenance dose for 3-4 days before surgery to allow the international normalized ratio to fall to around 1.5 at the time of the surgery. If the international normalized ratio is around 1.5 at the time of the procedure, warfarin will not cause hemorrhagic tendency and thrombus formation. This low-intensity warfarin doesn't interfere with surgical procedures, including abdominal surgery, orthopedic surgery, cardiac surgery, and tooth extraction. Further reduction of the dose of warfarin is dangerous and not necessary. Warfarin should not be stopped before the hair transplantation in patients at high risk for thrombosis.

Dabigatran (Pradaxa in the USA, Australia, and Europe,
Pradax in Canada, Prazaxa in Japan) is a new oral anticoagulant drug that is a direct thrombin inhibitor. Its bio-availability is not affected by foods, and similarly effective results are obtained without frequent blood tests of the international normalized ratio of prothrombin time. The United States Food and Drug Administration (FDA) approved Pradaxa on October 2010 for the prevention of stroke in patients with non-valvular atrial fibrillation. ${ }^{13-15}$ The maintenance dosage of dabigatran for anticoagulant therapy is $300 \mathrm{mg}$ per day. The dosage of dabigatran should be reduced to $220 \mathrm{mg}$ per day to avoid bleeding complication in patients over 70 years of age or in patients with moderately impaired renal function. The half-life of dabigatran is 12-17 hours. If you stop dabigatran for 24 hours before surgery, coagulation activity will return to normal at the time of the procedure. Major surgery is possible if you stop dabigatran for 2 days before surgery.

Other promising new oral anticoagulants include apixaban (Eliquis in Europe $)^{16}$ and rivaroxaban, which are selective direct inhibitors of clotting factor $\mathrm{Xa}$. The half-life of apixaban is 9-14 hours and of rivaroxaban 7-11 hours.

\section{Conclusion}

1. Antithrombotic therapy consists of a combination of anticoagulant drugs and antiplatelet drug. An update on the guidelines for the management of patients under antithrombotic therapy has recommended that these drugs should be continued before surgery in patients with a high risk of thrombosis.

2. The risk of bleeding and the risk of thrombosis should be compared in patients under antithrombotic therapy. The risk of thrombosis is low in patients with permanent atrial fibrillation. Patients with coronary artery stent and mechanical heart valve are at high risk of thrombosis resulting in cardiovascular events with high mortality. Anticoagulant and antiplatelet drugs should be continued before surgery in high-risk patients.

3. Within 12 months after percutaneous coronary intervention, coronary patients with a drug eluting stent require two types of antiplatelet drugs for the prevention of coronary artery stent thrombosis and the hair transplant operation should be postponed. In patients who underwent drug eluting stent implantation more than a year prior to transplantation, you should continue aspirin if you stop clopidogrel for 5 days. If you stop both drugs, you can stop them for 3 days but no longer. In patients more than 2 years after drug eluting stent implantation, you can stop aspirin for 3-5 days before surgery. Low-dose aspirin does not cause hemorrhagic tendency and it can be continued before hair transplantation.

4. Permanent atrial fibrillation is relatively safe, whereas paroxysmal atrial fibrillation is dangerous. In patients with atrial fibrillation, warfarin is commonly used as an anticoagulant drug. Low-intensity warfarin with an international normalized ratio of about 1.5 doesn't interfere with major surgery, and it can be continued before the hair transplantation.

\section{References}

1. Garcia Rodriguez, L.A., et al. Discontinuation of low dose aspirin and risk of myocardial infarction: case-control study in UK primary care. BMJ (Clinical research ed.). $19 \mathrm{Jul}$ 2011; 343:d4094. doi: 10.1136/bmj.d4094.

2. Pignone, M., et al. Aspirin for primary prevention of cardiovascular events in people with diabetes: a position statement of the American Diabetes Association, a scientific statement of the American Heart Association, and an expert consensus document of the American College of $\Rightarrow$ page 64 


\section{Guidelines for management}

from page 63

Cardiology Foundation. Circulation. 27 May 2010; DOI: 10.1161/CIR.0b013e3181e3b133.

3. Roger, V.L., et al. On behalf of the American Heart Association Statistics Committee and Stroke Statistics Subcommittee. Heart disease and stroke statistics- 2011 update: a report from the American Heart Association. Circulation. 2011; 123:459-463.

4. Taguchi, I., et al. Stent thrombosis after drug eluting stent implantation. Circ J. 2011; 75:1316-1317.

5. Nakagawa, Y. Speculative mechanisms for very late stent thrombosis after drug eluting stent implantation. Circ J. 2011; 75:779-780.

6. Connolly, S.J., et al. Dabigatran versus warfarin in patients with atrial fibrillation. $N$ Engl J Med. 2009; 361:1139-1151.

7. Wann, L.S., et al. 2011 ACCF/AHA/HRS focused updated on the management of patients with atrial fibrillation (update on dabigatran): a report of the American College of Cardiology Foundation/American Heart Association task force on practice guideline. Circulation. 2011; 123:1144-1150.

8. Cairns, J.A., et al. Canadian Cardiovascular Society atrial fibrillation guidelines 2010: prevention of stroke and systemic thromboembolism in atrial fibrillation and flutter. Can J Cardiol. 2011; 27:74-90.

9. Valentin, F., et al. ACC/AHA/ESC guidelines for the management of patients with atrial fibrillation: executive summary a report of the American College of Cardiology/American Heart Association task force on practice guidelines and the European Society of Cardiology Committee for practice guidelines and policy conference (committee to develop guidelines for the management of patients with atrial fibrillation) developed in collaboration with the North American Society of Pacing and Electrophysiology. Circulation. 2011; 104:2118-2150.

10. Ogawa, S., et al. Guidelines for pharmacotherapy of atrial fibrillation (Japanese Circulation Society 2008). Circ J. 2008; 72(Suppl.IV):1581-1658.

11. Mant, J., et al. Warfarin versus aspirin for stroke prevention in an elderly community population with atrial fibrillation (the Birmingham Atrial Fibrillation Treatment of the Aged Study, BAFTA): a randomized controlled trial. Lancet. 2007; 370:493-503.

12. Hirsh, J., et al. American Heart Association/American College of Cardiology Foundation guide to warfarin therapy. $J$ Am Coll Cardiol. 2003; 41:1633-1652.

13. Turpie, A.G., et al. New oral anticoagulants in atrial fibrillation. Eur Heart J. 2008; 29:155-165.

14. Connolly, S.J., et al. Dabigatran versus warfarin in patients with atrial fibrillation. $N$ Engl J Med. 2009; 361:1139-1151.

15. Eikelboom, J.W., et al. Risk of bleeding with 2 doses of dabigatran compared with warfarin in older and younger patients with atrial fibrillation: an analysis of the randomized evaluation of long-term anticoagulant therapy (RE-LY) trial. Circulation. 2011; 123:2363-2372.

16. Granger, C.B., et al. Apixaban versus warfarin in patients with atrial fibrillation. $N$ Engl J Med. 2011; 365:doi:10.1056/ NEJMoa1107039.

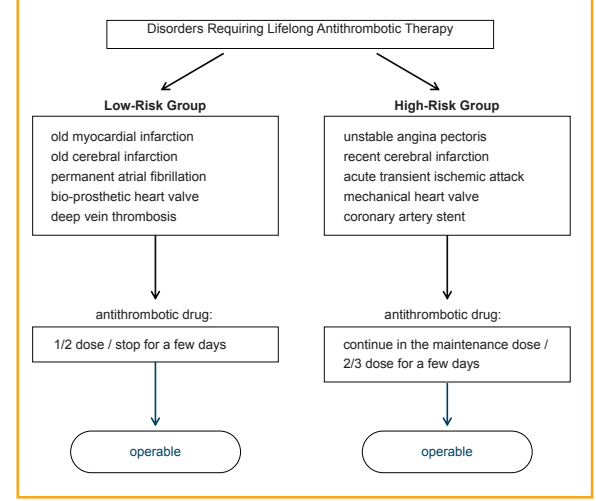

Flow Chart 1. Risk of Cardiovascular Events and Control of Antithrombotic Therapy

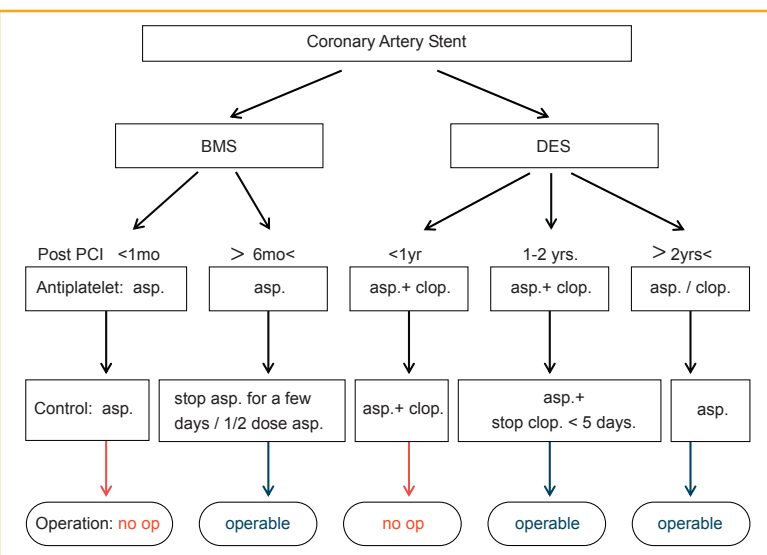

Abbreviations: BMS: bare metal stent, DES: drug eluting stent, PCl: percutaneous coronary intervention, asp.: aspirin $75-300 \mathrm{mg} /$ day, clop.: clopidogrel $75 \mathrm{mg} /$ day.

Flow Chart 2. Coronary Artery Disease and Antiplatelet Drug

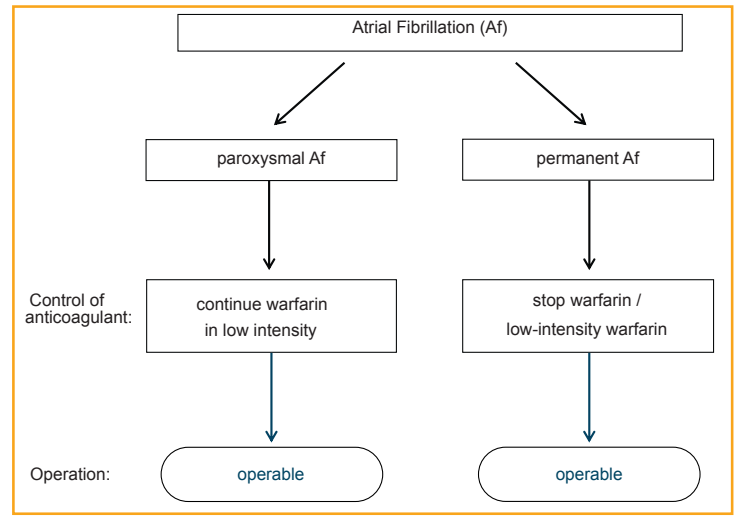

Flow Chart 3. Atrial Fibrillation and Anticoagulant Drug

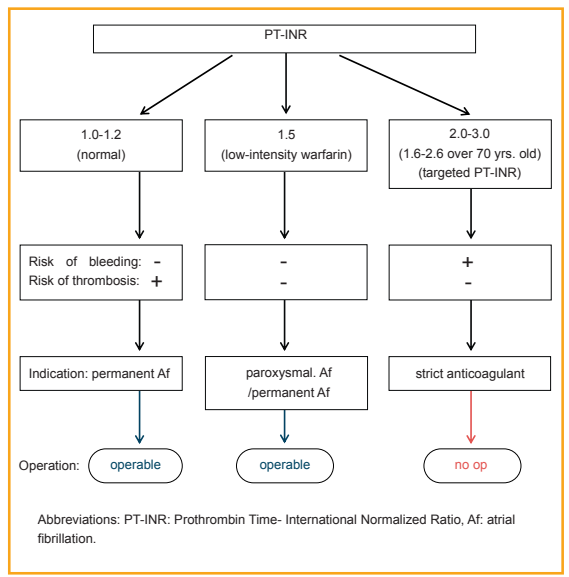

Flow Chart 4. Control of Anticoagulant in Atrial Fibrillation 